\title{
What's new on circulating tumor cells? A meeting report
}

\author{
Evi S Lianidou*, Dimitris Mavroudis², Georgia Sotiropoulou ${ }^{3}$ Sophia Agelaki ${ }^{2}$ and Klaus Pantel ${ }^{4}$
}

\begin{abstract}
Circulating tumor cells (CTCs) provide unique information for the management of cancer patients. The 7th International Symposium on Minimal Residual Cancer has focused on state of the art research, including exciting advances in understanding the biology of metastasis, CTCs and tumor dormancy. Particular emphasis was placed on the relationship of CTCs to cancer stem cells (CSCs) and the relevance of most recent findings for the development of new targeted therapies. CTCs were evaluated as promising tumor biomarkers and the design and results of the first clinical trials to determine their clinical utility were discussed together with state of the art technology platforms for CTC imaging, detection, quantification and molecular characterization. A liquid biopsy approach that can be used for prognostic and predictive purposes was proposed for the analysis of CTCS.
\end{abstract}

\section{Introduction}

Current research on circulating tumor cells (CTCs) present in the peripheral blood and disseminated tumor cells (DTCs) present in bone marrow provides new insight into the biology of tumor dormancy and tumor cell dissemination and will open new avenues for the early detection of metastatic spread and its successful treatment [1]. The 7th International Symposium on Minimal Residual Cancer, organized by Evi Lianidou, Dimitris Mavroudis, and Klaus Pantel, was held in Athens, Greece on 16 to 19 September 2009. The symposium brought together 220 basic and clinical researchers who presented cutting-edge research and had an excellent opportunity to have stimulating intense discussions on new technologies and clinical implications of CTC/

*Correspondence: lianidou@chem.uoa.gr

'Department of Chemistry, University of Athens, 15771 Athens, Greece

Full list of author information is available at the end of the article
DTC detection. We summarize in this report the major findings presented by invited speakers of this meeting.

\section{Biology of metastasis and tumor dormancy}

Danny R Welch (University of Alabama, Birmingham, AL, USA) presented a vivid account of metastasis suppressors, and especially breast cancer metastasis suppressor 1 (BRMS1), which is significantly downregulated in metastatic disease by epigenetic silencing [2] and coordinately regulates expression of multiple metastasis-associated microRNAs [3]. BRMS1 differentially attenuates cellular responses to mitogenic signals at varying steps within the same signaling cascade, and specific modulation of signaling responses received from the microenvironment may ultimately dictate which environments are permissive or restrictive for tumor cell growth $[3,4]$.

George Calin (MD Anderson Cancer Center, Houston, TX, USA) described the roles of microRNAs as metastasis activators or suppressors. His concept that the same microRNAs could be involved in both the CSC phenotype and the ability of specific cancer cells to give rise to metastases represents a mechanistic link between the initial and the final steps of tumorigenesis [5].

Jean-Paul Thiery (IMCB A STAR, Biopolis, Singapore) illustrated how epithelial-mesenchymal transition (EMT) can play a major role in local and distant dissemination of carcinomas. Understanding of the molecular mechanisms regulating EMT in solid tumors will provide new insights into mechanisms of cancer progression, detection of metastases, and development of effective and mechanismbased agents for improved therapeutic intervention [6].

Philip Tsichlis (Tufts University, Boston, MA, USA) highlighted how microRNAs differentially regulated by Akt isoforms control EMT and stem cell renewal in cancer cells. His data show that induction of EMT, stem cell renewal, cell survival and proliferation is controlled by microRNAs whose abundance depends on the balance between Akt1 and Akt2 rather than on the overall activity of Akt [7].

In a stimulating lecture Julio Aguirre-Ghiso (Mount Sinai School of Medicine, New York, NY, USA) reported on the roles of stress signaling and the microenvironment as regulators of tumor cell dormancy. Interestingly, 
dormancy of DTCs in the bone marrow may depend on the activation of tumor growth factor-beta signaling and p38 activation, p53 induction, and ERK inhibition in DHEp3 cells, indicating that microenvironment-derived signals that impinge on stress signaling pathways might be sufficient to induce a protracted state of cellular tumor dormancy [8].

Ann Chambers (University of Western Ontario, London, Ontario, Canada) presented a stimulating update on imaging of experimental cancer metastasis and tumor dormancy recapitulated in a three-dimensional cell culture system. Initiation of proliferation and maintenance of metastatic growth by a subset of cells delivered to secondary sites represent rate-limiting steps, but a larger population of cells that remain in a dormant state appears to be resistant to cytotoxic chemotherapy and retains metastasis-forming potential [9].

Lisa M Coussens (University of California, San Francisco, CA, USA) indicated that anti-tumor-acquired immunity mediated by $\mathrm{CD} 4^{+} \mathrm{T}$ lymphocytes may promote cancer by engaging cellular components of the innate immune system and identify $\mathrm{T}_{\mathrm{H}}$ 2-polarized $\mathrm{CD} 4{ }^{+}$ $\mathrm{T}$ lymphocytes as potential targets for anti-cancer therapy [10].

\section{Circulating tumor cells and cancer stem cells}

Subsets of CTCs or DTCs have a putative breast cancer stem-cell phenotype. New data on tumor stem and/or progenitor cells and their involvement in metastasis were presented. Bone marrow appears to be a special reservoir for DTCs [11]. Michael F Clarke (Stanford Institute for Stem Cell Biology and Regenerative Medicine, CA, USA), one of the leaders in the field of CSCs, gave a state of the art keynote lecture on the molecular analysis of normal and malignant epithelial stem cells. In his talk, Michael Clarke presented data on extensive molecular and cellular analyses of normal and malignant stem cells that have revealed new pathways that regulate self-renewal as well as resistance to cytotoxic therapies [12]. Maintenance of dormancy is critical for preservation of stem cell function; however, it is not clear if dormancy is in fact the interval (which may be long) between one cell division and the next, or represents a defined state in which stem cells actively enter and exit.

Andreas Trumpp (DKFZ, Heidelberg, Germany) referred to the identification of a long-term dormant population within mouse hematopoietic stem cells (HSCs) that bears the Lin-Sca ${ }^{+} \mathrm{CKit}^{+} \mathrm{CD} 150^{+} \mathrm{CD} 48^{-} \mathrm{CD} 34$ - phenotype. Dormant HSCs were shown to harbor the vast majority of multi-lineage long-term self-renewal activity and to be efficiently activated to self-renew in response to bone marrow injury or granulocyte colony-stimulating factor stimulation. After re-establishment of homeostasis, activated HSCs returned to dormancy, suggesting that
HSCs reversibly switch from dormancy to self-renewal under conditions of hematopoietic stress. One of the reasons CSCs are thought to escape anti-proliferative chemotherapy is their relative dormancy. Dr Trumpp showed that HSCs treated with interferon-alpha efficiently exit quiescence and are sensitized to chemotherapy drugs and that this effect is mediated by STAT1 or Sca-1. He also discussed the implications of these results for the design of strategies to target dormant chronic myeloid leukemia (CML) stem cells not targetable by imatinib alone [13].

Since CSCs survive standard cancer therapies and can theoretically regenerate the tumor even after killing the bulk of tumor cells, novel treatment strategies should eliminate CSCs in order to be effective. Signaling pathways that maintain CSCs represent attractive targets for these therapies. In his lecture, Hasan Korkaya (University of Michigan Comprehensive Cancer Center, Ann Arbor, MI, USA) reported that stem or progenitor cells in both normal breast tissue and breast tumors are dependent on the phosphatase and tensin homolog (PTEN)/phosphoinositide 3-kinase (PI3K)/Akt pathway for their continued growth. He also showed that perifosine, an Akt inhibitor, could specifically target the tumorigenic breast CSCs growing in mice. He concluded that these findings support the idea that selective targeting of breast CSCs through the PTEN/PI3K/Akt pathway may have important clinical implications in the treatment of patients with breast cancer [14].

\section{CTCs and DTCs as tumor biomarkers - clinical trials and therapeutics}

CTC-based platforms were shown to be effective for testing the efficacy of novel drugs. Howard I Scher (Memorial Sloan-Kettering Cancer Center, New York, NY, USA) described the application of CTCs as a 'liquid biopsy' in the evaluation of two therapies directed at specific molecular alterations that contribute to the progression of castration-resistant prostate cancer, the 17,20-lyase inhibitor abiraterone, and MDV3100, a novel anti-androgen [15].

Bernard Polzer (University of Regensburg, Germany) has shown that detection of cytokeratin-positive $\left(\mathrm{CK}^{+}\right)$ cells in bone marrow samples taken months to years after surgery had no impact on the survival of prostate cancer while the detection of epithelial cell adhesion moleculepositive $\left(\mathrm{EpCAM}^{+}\right)$cells in the bone marrow after surgery was predictive of metastatic relapse. Using single cell comparative genomic hybridization this group has demonstrated that $\mathrm{EpCAM}^{+}$cells harbored significantly more genomic aberrations than $\mathrm{CK}^{+}$cells, which may explain their high metastatic potential [16].

The prognostic significance of CTCs in early breast cancer was summarized by Dimitris Mavroudis 
(University of Crete, Heraklion, Greece). Prior to the administration of adjuvant chemotherapy, detection of CK19 mRNA-positive CTCs was associated with shorter disease-free survival (DFS) and overall survival (OS). Significantly decreased DFS and OS were observed for the post-chemotherapy CTC-positive patients. The prognostic utility of monitoring CTCs during adjuvant tamoxifen therapy was also shown in patients with hormone receptor-positive tumors since failure of tamoxifen to eradicate CTCs was associated with shorter DFS and OS. Immunofluorescent analysis of CTCs demonstrating the common expression of molecules such as phosphorylated focal adhesion kinase, phosphorylated PI3K, phosphorylated epidermal growth factor receptor, phosphorylated AKT, vascular endothelial growth factor and its receptor, and hypoxia-inducible factor- $1 \alpha$ in CTCs could be exploited for designing targeted therapies [17].

Human epidermal growth factor receptor 2 (HER2) status may be different between primary tumor and minimal residual disease as reflected by CTCs/DTCs according to Tanja Fehm (University of Tübingen, Germany). This may be due to either the acquisition of HER2 amplification during dissemination and tumor progression or due to a small subclone of HER2-positive cells in the primary tumor that has shed its tumor cells since the HER2-positive cells have a growth and survival advantage and can therefore be detected more frequently in bone marrow and blood. These results indicate that a substantial subset of HER2-negative breast cancer patients do not receive HER2-targeted therapy despite HER2-positive persistent tumor cells [18].

Bjorn Naume (The Norwegian Radium Hospital, Oslo, Norway) presented data showing that DTC status is an independent predictor of systemic relapse and breast cancer death at both diagnosis and follow-up. Initial data from the SATT study, which includes more than 1,100 breast cancer patients receiving non-taxane anthracyclinecontaining adjuvant chemotherapy, indicate that the DTC status changed with secondary chemotherapy in a significant proportion of patients.

Are DTCs or CTCs better for their predictive and prognostic value in breast cancer patients? This question was addressed by Jean Yves Pierga (Institute Curie, Paris, France). By summarizing data from eight published reports showing 66 to $94 \%$ concordance rates for DTC/ CTC detection, he concluded that DTC detection is of no clinical interest for metastatic breast cancer patients. However, for non-metastatic disease DTC detection constitutes an evidence-based level I prognostic factor and remains the reference method. On the contrary, Dr Pierga suggested that CTC detection has demonstrated prognostic and predictive value in metastatic breast cancer patients, while for early disease the level of evidence needs to be further substantiated.
Preliminary results of the SUCCESS trial were presented by Wolfgang Janni (Heinrich-Heine-Universität, Düsseldorf, Germany). CTCs of 3,658 early breast cancer patients at primary diagnosis and during adjuvant chemotherapy as well as endocrine and bisphosphonate treatment were analyzed using the CellSearch System (Veridex; Warren, NJ, USA). While the presence of CTCs before systemic treatment did not show prognostic relevance for DFS and OS, persistence of CTCs after chemotherapy was a significant predictor for both reduced DFS and OS. Dr Janni also pointed out that persisting CTCs after primary treatment may lead to an indication for extended adjuvant therapy, which needs to be investigated in future trials.

Massimo Cristofanilli (Fox Chase Cancer Center, Philadelphia, PA, USA) underscored that the prognostic value of CTCs using the CellSearch System in patients with metastatic breast cancer was independent of phenotype and has superior value compared to standard and functional imaging with [18F]fluorodeoxyglucose positron emission tomography [19]. Moreover, recent data indicate that CTCs represent a heterogeneous population that includes cells that have lost epithelial markers and have undergone EMT. Indeed, CTCs are characterized by higher expression of HER2 and loss of estrogen receptor expression compared to cancer cells isolated from the primary or metastatic tumors. Furthermore, additional phenotypic features suggest correlation with tumorinitiating cancer cells (for example, expression of Notch-1). Dr Cristofanilli speculated that a more global assessment of the microscopic disease may facilitate its use for monitoring and therapeutic targeting.

The challenges of using CTCs in clinical trials were addressed by Michalis Ignatiadis (Jules Bordet Institute, Brussels, Belgium). He pointed out that CTCs are already an integral part of ongoing studies such as the ALTTO trial in the adjuvant setting and the randomized SWOG 0500 study in metastatic disease, where the detection of CTCs at baseline and after the first cycle of front-line chemotherapy will guide treatment strategies. He also presented the outline of the 'TREAT CTC' trial, which will examine the role of trastuzumab as secondary adjuvant therapy in women with HER2-negative early breast cancer who have detectable HER2-positive CTCs.

Dave Hoon (John Wayne Cancer Institute, Santa Monica, CA, USA) reported on data from two international randomized phase III studies involving a melanoma vaccine in surgically resected stage III or IV melanoma patients where CTCs were detected at different time-points using optimized multimarker quantitative real-time RT-PCR. In both studies pretreatment detection of CTCs could provide significant prediction of DFS and OS in a multivariate analysis. These results indicate that CTC detection could be used 
for stratification of melanoma patients for better disease management.

Data from CTC studies in castration-resistant prostate cancer patients treated with abiraterone acetate, a highly specific CYP17 inhibitor, were presented by Gert Attard (Institute of Cancer Research, London, UK) [20]. A decline in CTC count of $\geq 30 \%$ during treatment with administration of abiraterone acetate was associated with a significant improvement in OS. Dr Attard suggested that circulating, non-apoptotic, nucleated $\mathrm{EpCAM}^{+} / \mathrm{CK}^{+} /$ CD45 cells are malignant in origin and that CTCs could be used to molecularly characterize castration-resistant prostate cancer.

\section{Novel technologies for the isolation, detection and characterization of CTCs}

Jonathan Uhr (University of Texas, Dallas, Texas, USA) presented a new technology for CTC characterization, based on a combination of hyper-spectral microscopic imaging and a microchip instrument using an immunemagnetic approach, to precisely quantify 17 different tumor markers in individual breast cancer cells from either touch preps of the primary tumor or CTCs in patients with tumor progression.

Leon Terstappen (University of Twente, Enschede, The Netherlands) presented improvements on the CellSearch System to detect more treatment targets on CTCs and stated that CTCs in blood of patients at primary diagnosis and after surgical intervention are quite different from CTCs in the metastatic setting. Molecular characterization of CTCs by fluorescence in situ hybridization has shown that most CTCs in hormone refractory prostate cancer are aneuploid, showing an extensive heterogeneity in the copy number of each of the chromosomes [21].

Richard Cote (Miller School of Medicine, Miami, FL, USA) presented a novel membrane microfilter device to isolate CTCs from blood with recovery rates of spiked tumor cells greater than $90 \%$ by exploiting size differences between tumor and normal blood cells. His group developed a quadruplex multimarker immunofluorescent assay that can be performed and evaluated directly on the microfilter device simultaneously, assessing CD44, CD24, ALD1 and cytokeratin using quantum dots as labels. The ability to efficiently capture CTCs on a small filter area and then analyze these cells on the same platform can substantially facilitate further detailed analysis of early and late events in metastasis.

According to Catherine Alix-Panabières (Hôpital SaintEloi - CHRU, Montpellier, France), who described the 'EPISPOT' assay, many patients harbor viable DTCs in bone marrow, even in cases with apparently localized tumors. In rare cases of breast cancer, CTCs can release HER2 as a potential mechanism to escape HER2-directed therapy [22], while in prostate cancer, a significant fraction of CTCs secreted fibroblast growth factor-2, a known stem cell growth factor.

Evi Lianidou (University of Athens, Greece) presented a novel real-time multiplex PCR assay for the simultaneous quantification of $C K-19, M A G E-A 3, H E R 2$ and $P B G D$ in CTCs of breast cancer patients, and a multiplexed PCR-coupled liquid bead array for the simultaneous detection of CK-19, HER2, Mammaglobin, MAGE-A3, TWIST-1 and PBGD as a control, in a very limited amount of sample, thus saving sample volume and reducing the total cost and time of analysis.

Morphologic and molecular characterization of CTCs at the single cell level were discussed in the context of individual patient management by Peter Kuhn (The Scripps Research Institute, La Jolla, CA, USA) and CTC analysis results from a small series case study in nonsmall cell lung cancer patients were compared with clinical progression.

Carl Wittwer (University of Utah, Salt Lake City, UT, USA) highlighted the importance of real-time PCR in the quantitative assessment of specific transcripts in minimal residual disease monitoring and described recent advances in high resolution melting analysis for variant scanning and genotyping.

\section{Conclusions and future perspectives}

In his key note lecture Klaus Pantel (University of Hamburg, Germany), who is the founder of this international meeting series, pointed out that detection of CTCs/DTCs may contribute to early cancer detection and provide independent prognostic information. Moreover, monitoring of CTCs/DTCs may become a biomarker for prediction of therapeutic efficacy in cancer patients, and CTC/DTC analyses may improve stratification of patients in need of (neo)adjuvant therapies. In this context, characterization of CTCs/DTCs opens a new avenue for identification of therapeutic targets and understanding resistance to therapies. Last but not least, research on CTCs/DTCs will contribute to a better understanding of the biology of metastatic development in cancer patients. The functional relevance of these findings can then be cross-validated in appropriate animal models. If the few CTCs/DTCs found in human blood or bone marrow samples grow in mice, it would be almost a proof of their stem cell nature.

The upcoming 8th International Symposium on Minimal Residual Cancer will be held in Osaka, Japan from 21 to 23 September 2011, organized by Professor Masaki Mori (Osaka University).

\section{Abbreviations}

BRMS1 = breast cancer metastasis suppressor 1; CSC = cancer stem cell; CTC = circulating tumor cell; DFS = disease-free survival; DTC = disseminating tumor cell; EMT = epithelial-mesenchymal transition; HER = human epidermal 
growth factor receptor; $\mathrm{HSC}=$ hematopoietic stem cell; OS = overall survival; $\mathrm{PI3K}=$ phosphoinositide 3-kinase.

\section{Author details}

'Department of Chemistry, University of Athens, 15771 Athens, Greece. 2Department of Medical Oncology, University Hospital of Heraklion, School of Medicine, University of Crete, 71110 Heraklion, Greece. ${ }^{3}$ Department of Pharmacy, University of Patras, Rion-Patras, 26500, Greece. ${ }^{4}$ Institute of Tumor Biology, University Medical Center Hamburg-Eppendorf, Martinistrasse 52, 20246 Hamburg, Germany.

\section{Competing interests}

The authors declare that they have no competing interests.

\section{Acknowledgements}

We would like to thank all participants for a wonderful meeting, and we are grateful to all companies that sponsored this meeting. We apologize to those participants whose work has not been mentioned owing to space constraints. A complete report on this meeting as well as supplementary data and a complete listing of speakers and presentation titles are available at the International Symposium on Minimal Residual Cancer web site [23].

Published: 23 July 2010

\section{References}

1. Pantel K, Alix-Panabières C, Riethdorf S: Cancer micrometastases. Nat Rev Clin Oncol 2009, 6:339-351.

2. Metge BJ, Frost AR, King JA, Dyess DL, Welch DR, Samant RS, Shevde LA: Epigenetic silencing contributes to the loss of BRMS1 expression in breast cancer. Clin Exp Metastasis 2008, 25:753-763.

3. Hurst DR, Edmonds MD, Scott GK, Benz CC, Vaidya KS, Welch DR: Breast cancer metastasis suppressor 1 up-regulates miR-146, which suppresses breast cancer metastasis. Cancer Res 2009, 69:1279-1283.

4. Vaidya KS, Harihar S, Phadke PA, Stafford L, Hurst DR, Hicks DG, Casey G, DeWald DB, Welch DR: Breast cancer metastasis suppressor-1 differentially modulates growth factor signaling. J Biol Chem 2008, 283:28354-28360.

5. Nicoloso MS, Spizzo R, Shimizu M, Rossi S, Calin GA: MicroRNAs - the micro steering wheel of tumour metastases. Nat Rev Cancer 2009, 9:293-302.

6. Thiery JP, Sleeman JP: Complex networks orchestrate epithelialmesenchymal transitions. Nat Rev Mol Cell Biol 2006, 7:131-142.

7. Iliopoulos D, Polytarchou C, Hatziapostolou M, Kottakis F, Maroulakou IG, Struhl K, Tsichlis PN: MicroRNAs differentially regulated by Akt isoforms control EMT and stem cell renewal in cancer cells. Sci Signal 2009, 2:62.

8. Adam AP, George A, Schewe D, Bragado P, Iglesias BV, Ranganathan AC, Kourtidis A, Conklin DS, Aguirre-Ghiso JA: Computational identification of a p38SAPK-regulated transcription factor network required for tumor cell quiescence. Cancer Res 2009, 69:5664-5672.

9. Hedley BD, Chambers AF: Tumor dormancy and metastasis. Adv Cancer Res 2009, 102:67-101.

10. DeNardo DG, Barreto JB, Andreu P, Vasquez L, Tawfik D, Kolhatkar N, Coussens LM: CD4(+) T cells regulate pulmonary metastasis of mammary carcinomas by enhancing protumor properties of macrophages. Cancer Cell 2009, 16:91-102.

11. Pantel $\mathrm{K}$, Brakenhoff $\mathrm{RH}$, Brandt B: Detection, clinical relevance and specific biological properties of disseminating tumour cells. Nat Rev Cancer 2008, 8:329-340.
12. Shimono Y, Zabala M, Cho RW, Lobo N, Dalerba P, Qian D, Diehn M, Liu H, Panula SP, Chiao E, Dirbas FM, Somlo G, Pera RA, Lao K, Clarke MF: Downregulation of miRNA-200c links breast cancer stem cells with normal stem cells. Cell 2009, 138:592-603.

13. Essers MA, Offner $S$, Blanco-Bose WE, Waibler Z, Kalinke U, Duchosal MA, Trumpp A: IFNalpha activates dormant haematopoietic stem cells in vivo. Nature 2009, 458:904-908.

14. Korkaya H, Paulson A, lovino F, Wicha MS: HER2 regulates the mammary stem/progenitor cell population driving tumorigenesis and invasion. Oncogene 2008, 27:6120-6130.

15. Scher HI, Jia X, de Bono JS, Fleisher M, Pienta KJ, Raghavan D, Heller G: Circulating tumour cells as prognostic markers in progressive, castrationresistant prostate cancer: a reanalysis of IMMC38 trial data. Lancet Oncol 2009, 10:23323-23329.

16. Weckermann D, Polzer B, Ragg T, Blana A, Schlimok G, Arnholdt H, Bertz S, Harzmann R, Klein CA: Perioperative activation of disseminated tumor cells in bone marrow of patients with prostate cancer. J Clin Oncol 2009, 27:1549-1556.

17. Kallergi G, Markomanolaki H, Giannoukaraki V, Papadaki MA, Strati A, Lianidou ES, Georgoulias V, Mavroudis D, Agelaki S: Hypoxia-inducible factor-1alpha and vascular endothelial growth factor expression in circulating tumor cells of breast cancer patients. Breast Cancer Res 2009, 11:R84.

18. Fehm T, Hoffmann O, Aktas B, Becker S, Solomayer EF, Wallwiener D, Kimmig R, Kasimir-Bauer S: Detection and characterization of circulating tumor cells in blood of primary breast cancer patients by RT-PCR and comparison to status of bone marrow disseminated cells. Breast Cancer Res 2009, 11:R59.

19. De Giorgi U, Valero V, Rohren E, Dawood S, Ueno NT, Miller MC, Doyle GV, Jackson S, Andreopoulou E, Handy BC, Reuben JM, Fritsche HA, Macapinlac HA, Hortobagyi GN, Cristofanilli M: Circulating tumor cells and [18F] fluorodeoxyglucose positron emission tomography/computed tomography for outcome prediction in metastatic breast cancer. J Clin Oncol 2009, 27:3303-3311.

20. Attard G, Reid AH, A'Hern R, Parker C, Oommen NB, Folkerd E, Messiou C, Molife LR, Maier G, Thompson E, Olmos D, Sinha R, Lee G, Dowsett M, Kaye SB, Dearnaley D, Kheoh T, Molina A, de Bono JS: Selective inhibition of CYP17 with abiraterone acetate is highly active in the treatment of castrationresistant prostate cancer. J Clin Oncol 2009, 27:3742-3748.

21. Swennenhuis JF, Tibbe AG, Levink R, Sipkema RC, Terstappen LW: Characterization of circulating tumor cells by fluorescence in situ hybridization. Cytometry A 2009, 75:520-527.

22. Alix-Panabières C, Vendrell JP, Slijper M, Pellé O, Barbotte E, Mercier G, Jacot W, Fabbro M, Pantel K: Full-length cytokeratin-19 is released by human tumor cells: a potential role in metastatic progression of breast cancer. Breast Cancer Res 2009, 11:R39.

23. International Symposium on Minimal Residual Cancer [http://www. ismrc2009.chem.uoa.gr]

doi:10.1186/bcr2601

Cite this article as: Lianidou ES, et al:: What's new on circulating tumor cells? A meeting report. Breast Cancer Research 2010, 12:307. 